\title{
Mining Millions of Metaphors
}

\author{
Brad Pasanek \\ English Department \\ Pomona College \\ West Sixth Street \\ Claremont, CA 91711 USA \\ BRAD.PASANEK@POMONA.EDU \\ D. Sculley \\ Department of Computer Science \\ Tufts University \\ 161 College Ave. \\ Medford, MA 02155 USA \\ DSCULLEY@CS.TUFTS.EDU
}




\begin{abstract}
One of the first decisions made in any research concerns the selection of an appropriate scale of analysis - are we looking out into the heavens, or down into atoms? To conceive a digital library as a collection of a million books may restrict analysis to only one level of granularity. In this paper we examine the consequences and opportunities resulting from a shift in scale, where the desired unit of interpretation is something smaller than a text: it is a keyword, a motif, or a metaphor. A million books distilled into a billion meaningful components become raw material for a history of language, literature, and thought that has never before been possible. While books herded into genres and organized by period remain irregular, idiosyncratic, and meaningful in only the most shifting and context-dependent ways, keywords or metaphors are lowest common denominators. At the semantic level - the level of words, images, and metaphors - long term regularity and patterns emerge in collection, analysis, and taxonomy. This paper follows the foregoing course of thought through three stages: first, the manual curation of a high quality database of metaphors; second, the expansion of this database through automated and humanassisted techniques; finally, the description of future experiments and opportunities for the application of machine learning, data mining, and natural language processing techniques to help find patterns and meaning concealed at this important level of granularity.
\end{abstract}


But the greatest thing by far is to be a master of metaphor. It is the one thing

that cannot be learnt from others.

- Aristotle, Poetics 1459a 4-5

\section{Introduction}

Considering the evolution of digital libraries, Gregory Crane (2006) asks the framing question, 'What do you do with a million books?" One of his conclusions is that digital libraries allow analysis at different levels of granularity or scale. And one important level of granularity is that of the individual figure, or metaphor. Decomposed into a billion meaningful components, a million books serve as raw material for bold new historical explorations of rhetoric, literature, and thought across genres and between books. In this paper, we examine the consequences of taking metaphors as our basic units of interpretation and detail the opportunities presented in so doing. We consider the tacit assumption, shared by a variety of scholars, that changing metaphors are indicative of broader cultural change. Viewing the history of literature at the semantic level, as a movement of metaphors through discourse, we may perceive long-term regularities in the flux of authors, texts, and genre. By checking the generalizations of literary history against collections of electronic texts, our work with metaphors delivers a host of surprising observations - both local and general.

In this paper, we introduce a database of metaphors of mind in eighteenth-century literature. This initial database is a hand-curated archive of figures of speech, and its contents were collected by keyword searching a variety of electronic text collections. Whereas the contents of the database were originally enlisted in the writing of a not untraditional project in the field of literary and intellectual history, it now serves as a small digital library of metaphors, one which not only enables additional traditional scholarly research but also a range of automated analysis techniques from the fields of machine learning and pattern recognition. 
In the remainder of this paper, we explore several key issues surrounding this collection. We review the manual construction of this initial collection in Section 2, and explain why we have chosen to focus on metaphors of the mind in particular. Section 3 explores results of machine learning classification of metaphors, which not only give promise for automating the expansion of this collection, but also yield new literary-analytical insights. The utility of working with this collection is demonstrated in Section 4, which reports a case study disrupting the intellectual history of the blank slate, a metaphor associated with John Locke's philosophy of mind. Section 5 extends this work by exploring the use of automated methods of analysis from machine learning and pattern recognition. The final section concludes with a discussion centered on plans for future work.

\section{A Metaphorical Database of the Mind}

Our initial collection, titled The Mind is a Metaphor, is currently composed of 8,741 metaphors of mind that have been harvested principally from collections of electronic texts (Pasanek, 2006). We treat the discourse of the British eighteenth century at large and draw upon philosophy, poetry, novels, plays, and all manner of prose pieces. We deal in those images - blank page, dark cave, empire, garden, mirror, room - that have long been associated with the mind and its workings. In doing so we hope to limn the structure of mental activity as it is figured in the literature of the long eighteenth century (1660-1830). Our approach in constructing this collection is digital, but our efforts remain continuous with the longer traditions of philology and intellectual history.

\subsection{Manually Harvesting Metaphors}

In this section, we review the manual methodology used to collect the initial set of metaphors. The Mind is a Metaphor database was first built by keyword searching the Chadwyck-Healey databases, Eighteenth Century Collections Online, Early English Books Online, and other

collections of electronic texts ${ }^{1}$. In Chadwyck-Healey alone, evidence was gathered from 877 books of poetry by 247 different poets, 96 long prose narratives, and 628 plays by 137 
different playwrights. The process of harvesting metaphors was labor intensive, requiring years of hand-labeling by a human expert (Pasanek, 2006). Indeed, the expertise called upon when dropping down into the middle of a thousand page expanse of text and deciding in a hermeneutic instant if a string of words contains a metaphor involves a traditional kind of training in rhetoric, literary theory, and intellectual history.

Pasanek (2006) worked through various iterations of a manual search protocol for harvesting metaphors. The first protocol searched for occurrences of the terms 'mind,' 'heart,' 'soul,' and a related set of words in a set of canonical and non-canonical texts, which revealed the full diversity of eighteenth-century metaphors of mind in anthologies of poetry, works of philosophy, obscure novels, journalism, and drama. The second iteration of the protocol included more refined searches for specific terms and phrases that signal the appearance of metaphors in a text. The third protocol added the refinement of proximity searches, which allowed collection of material in a more focused, efficient manner. In most cases, the keyword search was exhaustive within the given literature database, finding all occurrences of a word or words (for example, 'mind' and 'eye' within a span of one hundred characters) were consulted before final decisions were made about the metaphoricity of any one sentence in which the terms in question appeared. Furthermore, the search often revealed additional metaphors and keywords in neighboring sentences, which were then added to the protocols, ultimately producing a mapped network of related metaphors. This effort has produced a curated data set of high quality, which can enable machine learning methods to automate both the expansion of this collection and the analysis of its contents.

\subsection{The Current Collection}

In October of 2005, the database that houses the collection of metaphors was published online at http://metaphorized.net. It is our hope that scholars from a wide range of disciplines - including cognitive linguists, intellectual historians, and philosophers - will find the massive library of metaphors both provocative and useful as a work of reference. As of today, the database contains over 8,600 records; each record or entry identifies and 
categorizes a single metaphor. Each of these metaphors is displayed in the context of the work in which it appears; it is taxonomized, identified by author, by date of publication, genre, rhetorical figure type, metaphor category, and so forth.

We know of three other open and online databases of metaphor of interest: John Barnden's databank, ${ }^{2}$ the Hamburg Metaphor Database, ${ }^{3}$ and The Conceptual Metaphor server. ${ }^{4}$ Barnden's databank is closest to our own collection of metaphors as it exclusively contains examples of figurative descriptions of mental states and processes ${ }^{5}$. Both Barnden's databank and the Hamburg Metaphor Database project acknowledge Lakoff's and Johnson's theory of conceptual metaphor as the paradigm that informs their collection and storage of metaphors (Lakoff and Johnson, 1980), and it is Lakoff's Conceptual Metaphor Home Page that provides the fastest introduction to the system of categorization employed by these cognitive linguists. Our collection differs from these other collections both in scale, being nearly an order of magnitude larger, and in scope. Our work is geared towards the history of metaphor and thought.

In writing the history of metaphor, the historian must pay particular attention to how key terms are used, how conceptual pictures are invoked, how the metaphors are employed, and by whom. The philosopher J. L. Austin (1961) has called this kind of effort (although only half-seriously) 'linguistic phenomenology'. One might compare a similar pronouncement of philologist E. R. Curtius (1990), who in all seriousness frames his own research questions in European Literature and Latin Middle Ages as a 'prolegomena to what I should like to call a phenomenology of literature' (ix). Attending to the metaphor of mind first, detailing its use and application in the context, we gain deeper insight into the function of metaphor at large. But by searching electronic texts, collecting metaphors in a database, and using techniques from computational linguistics to analyze the assembled material, the linguistic phenomenologist can multiply examples well beyond what Austin or Curtius would have believed possible. 


\subsection{The Virtues of Drudgery}

In his 1755 Dictionary of the English Language, Samuel Johnson is careful to define 'lexicographer' as 'writer of dictionaries; a harmless drudge.' That adjective 'harmless' is tongue in cheek-or something more. Drudgery is wearisome, yes, but also transformative, intoxicating, and empowering. Drudgery is at the heart of the interdisciplinary conversation in which literary historians and computer scientists participate, because the computer is the most thorough of drudges. The literary historian and the computer scientist come together to develop methods for automating the very drudgery that characterizes lexicography itself. The humanist must put aside his fears of machines run amok, even as he remembers that Victor Frankenstein's creature was also weaned on Milton.

\section{Learning to Recognize Metaphors}

The process of manually searching for and tagging metaphors is painstaking, costly work, but the efforts of the literary critic have produced a high quality database. From a literary point of view, this database is a library of metaphors, but from a machine learning perspective, this database is a rich set of training data for creating automatic classifiers that can automatically tag metaphors and non-metaphors. This is the classic machine learning framework. A classifier is first trained on a set of known, labeled data, and is then asked to classify a large set of new data for which the true labels are not yet known (Mitchell, 1997).

In this section, we demonstrate the feasibility of a machine learning approach to metaphor recognition by training a classifier to distinguish metaphors from non-metaphors in the works of several eighteenth-century authors. We find not only that our classifiers are able to distinguish metaphorical usage within a single author's work, but also that a model learned on one author often transfers to other authors. For example, Shakespeare's Renaissance metaphors inform the discovery of the Augustan metaphors in Pope's poetry, and vice versa. This finding has practical value as a method of bootstrapping the manual effort of labeling metaphors. We might add, these findings trouble a traditional belief, first articulated in Aristotle's Poetics, that the use and recognition of metaphors cannot be learned. 
In the remainder of this section, we describe our methodology. We review the standard practice in text classification and show how we represent each of our metaphors with a bag of words vector. This vector data is used to train a classifier called a support vector machine (SVM), which is known to give state of the art performance on the related task of text classification (Joachims, 1998). We then detail our experimental methodology, and report results showing classification accuracy, learning rates, and transfer.

\subsection{Mapping Metaphors to Vectors}

In machine learning, as in classical information retrieval, it is standard practice to represent text documents as vectors (Mitchell, 1997; Salton and Buckley, 1988). This enables the use of a variety of machine learning methods originally designed for vector-based data, including SVM classifiers described below. The vector representation most commonly used is the bag of words vector. A bag of words vector $\vec{x}$ exists in a vector space $R^{m}$. This is a space of very high dimensionality, in which $m$ represents the total number of unique words in the vocabulary, and each word $i$ is represented by a unique coordinate $\vec{x}_{i}$.

We can map a text $t$ to a vector $\vec{x}$ in the space $R^{m}$ by letting $\vec{x}_{i}=c_{i}(t)$, where $c_{i}(t)$ shows the number of occurrences of word $i$ in the text $t$. This simple count based scheme for scoring words is the one that we employ; other scoring schemes include binary scoring and the TF-IDF scoring method (Salton and Buckley, 1988). The mapping may be accomplished with low computation and memory cost by exploiting sparsity of the data: only a small fraction of all possible vocabulary words are present in most texts. Sparse data structures exist which do not require any $\vec{x}_{i}=0$ to be represented. In general, we assume that all punctuation has been removed, and all letters converted to lower case. However, we do not perform word stemming, owing to irregularities in eighteenth-century spelling. ${ }^{6}$

In our domain of metaphors of the mind, body, heart, and soul, we start with a database of text excerpts. Each excerpt consists of a small number words - usually no more than a sentence - containing a keyword (such as "mind") and its context. Each excerpt has been labeled as being a metaphor, or a non-metaphor. (See Figure 1.) We create our data set 


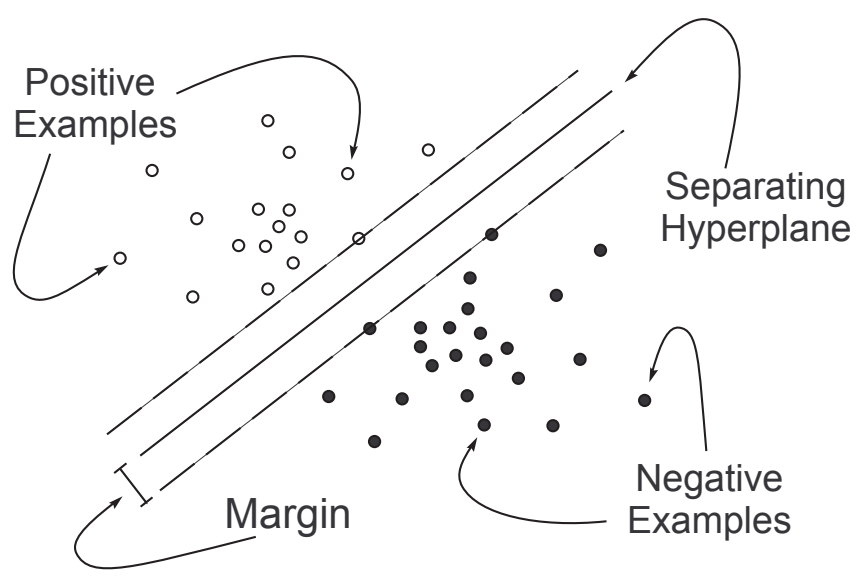

Figure 1: Visualizing SVM Classification. An SVM learns a hyperplane that separates the positive and negative data examples with the maximum possible margin. In our case, positive examples are metaphors, and negative examples are non-metaphors.

treating each excerpt as a unique text $t$ which is mapped to a bag of words vector $\vec{x}$, and is given label $y$. In this domain, positive examples of metaphors are given label $y=(+1)$ and negative examples (i.e., non-metaphors) are given label $y=(-1)$. Thus, the database of metaphors is mapped to a set of labeled vectors, with which we can train and test our classifiers.

\subsection{Classification with Support Vector Machines (SVMs)}

In this set of experiments, we work with linear SVM classifiers. SVMs are one form of linear classifier, and give state of the art performance on text classification (Joachims, 1998). They are particularly well suited to the task of learning high dimensional data, such as our data set of sparse, high dimensional bag of words vectors.

Recall that our data of metaphors and non-metaphors are represented as vectors, which can be thought of as points in a space. A linear SVM finds a hypothesis vector $\vec{w}$ and bias term $b$, which define a hyperplane separating two classes of data - in our case, separating metaphors from non-metaphors (see Figure 1). While there are many machine learning 
methods that create a separating hyperplane (Mitchell, 1997), SVM are statistically robust because they find a hyperplane that maximizes the margin between the two classes of data. This is formalized by finding a hypothesis vector $\vec{w}$ which minimizes the following objective function:

$$
\tau(\vec{w}, \xi)=\frac{1}{2}\|\vec{w}\|^{2}+C \sum_{i=1}^{m} \xi_{i}
$$

Minimizing the above function has two goals. Minimizing the right hand term corresponds to minimizing training error. Each value $\xi_{i}$ shows the amount of error the current hypothesis gives on the corresponding example in the training data $\mathbf{x}_{i}$, and $\sum_{i=1}^{m} \xi_{i}$ is a summation of all the training errors. Clearly, effective training should minimize error on the training data. Minimizing the left hand term, $\frac{1}{2}\|\vec{w}\|^{2}$, corresponds to minimizing the complexity of the model. That is, SVMs prefer a simple hypothesis to a complex hypothesis. Intuitively, a simple hypothesis is one with a large margin around the hypothesis separating the two classes (see Figure 1). The goals of minimizing training error and minimizing model complexity are often in tension; the parameter $C$ is set by the user to tell the algorithm how much weight to give to each of these goals in training (Scholkopf and Smola, 2002).

This idea of minimizing training error while keeping the model as simple as possible puts a guarantee on the ability of the classifier to generalize its findings and classify new (previously unseen) examples well. For a complete overview of support vector machines, see the introductory tutorial by Burges (1998), or texts by Cristianini and Shawe-Taylor (2000) and Scholkopf and Smola (2002). In all of our classification experiments, we used the LIBSVM implementation (Chang and Lin, 2001), with linear kernel, at default settings.

\subsection{Testing Recognition of Metaphorical Usage}

We first tested whether metaphors and non-metaphors are distinguishable within a single author's work. For these experiments, we used SVM classification, and used 100-fold cross validation to secure results. ( $n$-Fold cross validation is a commonly used method of establishing test error rates, in which the data set is randomly divided into $n$ distinct subsets. A total of $n$ tests are then run; for each test $n-1$ sets are used for training, 
Table 1: Results on Recognition of Metaphorical Usage. Results are given for each of the six author data sets, evaluated using 100-fold cross validation, and also for a data set of all authors tested together, also evaluated using 100-fold cross validation. The number of metaphors and non-metaphors in each data set are also given.

\begin{tabular}{|c|c|c|c|c|c|c|c|c|c|}
\hline & COWPER & FIELDG. & KEATS & LONSD. & Milton & POPE & RCHDSN. & SKSPR. & ALL \\
\hline $\begin{array}{l}\text { MPHRs. } \\
\text { NonMphrs. }\end{array}$ & $\begin{array}{l}130 \\
219\end{array}$ & $\begin{array}{c}133 \\
63\end{array}$ & $\begin{array}{c}75 \\
100\end{array}$ & $\begin{array}{l}168 \\
222\end{array}$ & $\begin{array}{c}34 \\
125\end{array}$ & $\begin{array}{c}190 \\
81\end{array}$ & $\begin{array}{c}159 \\
98\end{array}$ & $\begin{array}{l}226 \\
724\end{array}$ & $\begin{array}{l}1115 \\
1632\end{array}$ \\
\hline ACCURACY & .885 & .878 & .869 & .936 & .862 & .919 & .848 & .944 & .867 \\
\hline
\end{tabular}

and the remaining set is used for testing (Witten and Frank, 2005).) We ran experiments on seven authors: William Cowper, Henry Fielding, John Keats, John Milton, Alexander Pope, Samuel Richardson, and William Shakespeare. Additionally, we ran experiments on the 'Lonsdale' data set, which contains metaphors collected from an important anthology of eighteenth-century women poets edited by Roger Lonsdale (1989). The data sets were of differing size and class distribution, as detailed in Table 1. Our results on these experiments, reported in Table 1, were encouraging. We achieved high levels of accuracy on all data sets, even those of relatively small size.

Learning Curves. These tests give us confidence that SVM classification may indeed be a plausible method for automatically harvesting more metaphors for 'The Mind is a Metaphor' database from the eighteenth century as well as from neighboring historical periods. But how much more manual labor must be conducted, especially in unexplored periods, before the application of machine learning methods can be effective? To answer this question, we plotted a set of learning curves for each data set, which appears in Figure 2. The learning curve plots training set size versus test accuracy. We constructed this plot averaging over 20 independent trials. The curve shows that, in general, high levels of accuracy may be obtained with relatively few total training examples. Most of the curves have hit their maximum performance with between 100 and 200 training examples. Thus, to expand our inquiry into the metaphorics of new periods, moving outward from the core 


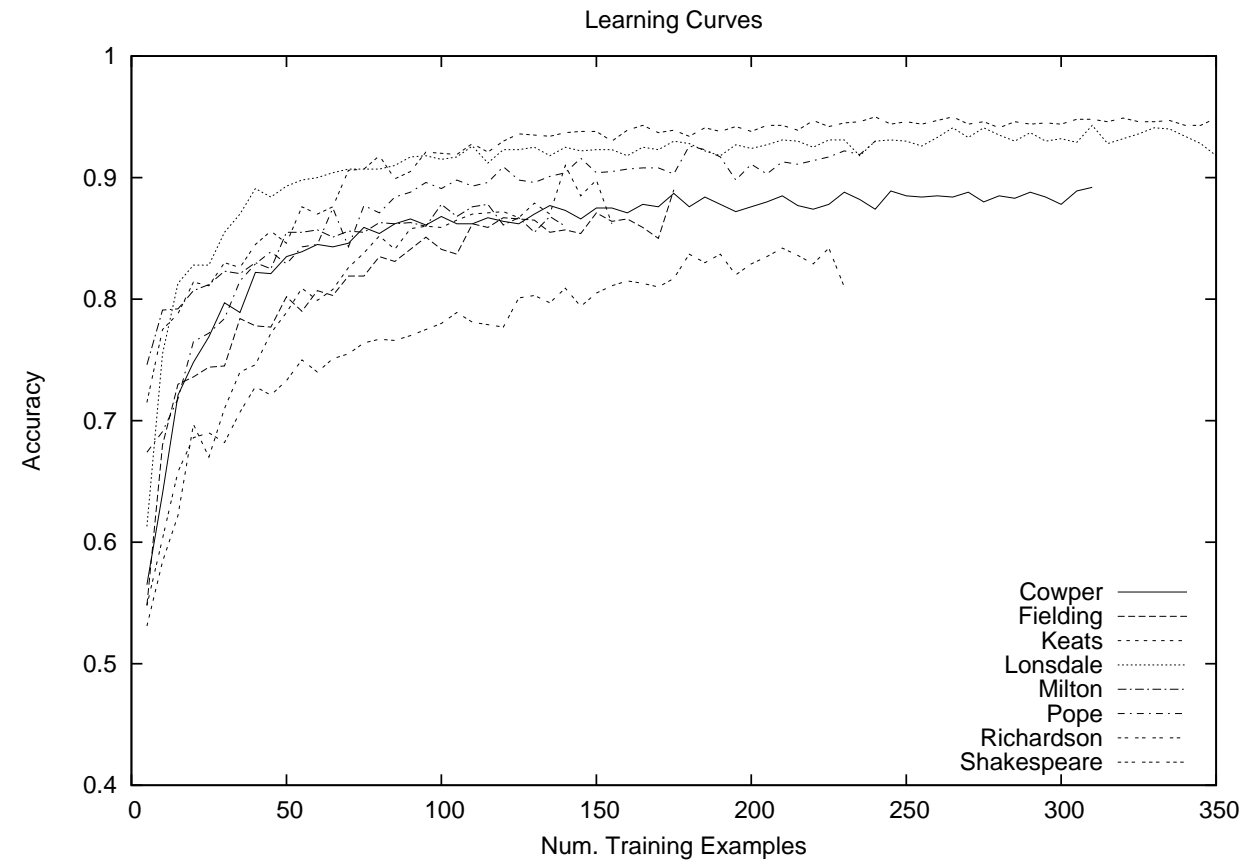

Figure 2: Learning Curves for Recognition of Metaphorical Usage. This graph shows how the accuracy of the classifier improves with the number of training examples, for each of the eight data sets. Note that a relatively small number of training examples is sufficient to achieve near-asymptotic accuracy. 
eighteenth-century examples by pushing forward into the future and reaching backward into the past - into the Renaissance and Victorian periods, respectively - may not necessitate any more than a small seed set of examples. In less than a week's time, an intimate cohort of period specialists could produce several such training sets for the long durée of mental metaphorics that stretches from medieval literature to present-day postmodern publications, which means we may well come to include new authors or genres and periods, without having to label many thousands of metaphors by hand.

\subsection{Testing Transfer}

Is it necessary to train a unique model for each individual author? We would hope that our models of metaphors and non-metaphors can generalize beyond a specific author. As a first test of this idea, we combined all eight data sets into one aggregate data set, and performed 100-fold cross validation on it. If the use of metaphors and non-metaphors does not generalize from author to author, we would expect that this aggregation would cause a significant decrease in classification accuracy. However, as the ALL column in Table 1 shows, SVM classification still achieved high levels of accuracy on this test.

We then explicitly tested the ability of models trained on one author to classify metaphors and non-metaphors from different authors. We did this by training a unique SVM classifier for each of the eight authors, and then testing that classifier on each of the remaining seven authors. Results of this experiment are given in Table 2, and are generally positive - models trained on many of the authors do indeed transfer to other authors. Notable exceptions include poor performance transferring between the novelists and poets and between certain poets and Shakespeare.

The results are surprisingly strong and enable an original path of analysis between authors. But how is it possible that a machine can use metaphors from Pope's neoclassical poetry to find metaphors in Shakespeare's playful, punning comedies? Pope and Shakespeare occupy two distinct, clearly distinguished periods in the history of literature; their writing is separated by a full century of turmoil and innovation. In survey courses under- 
Table 2: Results on Transfer of Metaphorical Recognition. Results are given for transfer of recognition accuracy. Model was trained on data set from one author; this model was tested on data set from other authors. Reported score reflects accuracy on test of distinguishing metaphors from non-metaphors.

\begin{tabular}{|c|c|c|c|c|c|c|c|c|}
\hline Trained ON & COWPER & FIELDG. & KEATS & $\begin{array}{l}\text { TeSTED On } \\
\text { LONSD. }\end{array}$ & Milton & POPE & RCHDSN. & SKSPR. \\
\hline $\begin{array}{l}\text { COWPER } \\
\text { FIELDING }\end{array}$ & $\begin{array}{c}\mathrm{X} \\
.590\end{array}$ & $\begin{array}{c}.648 \\
\mathrm{X}\end{array}$ & $\begin{array}{l}.794 \\
.651\end{array}$ & $\begin{array}{l}.592 \\
.892\end{array}$ & $\begin{array}{l}.736 \\
.836\end{array}$ & $\begin{array}{l}.708 \\
.705\end{array}$ & $\begin{array}{l}.525 \\
.728\end{array}$ & $\begin{array}{l}.623 \\
.768\end{array}$ \\
\hline $\begin{array}{r}\text { KEATS } \\
\text { LONSDALE }\end{array}$ & $\begin{array}{l}.871 \\
.734 \\
\end{array}$ & $\begin{array}{l}.694 \\
.837\end{array}$ & $\begin{array}{c}\mathrm{X} \\
.606 \\
\end{array}$ & $\begin{array}{c}.915 \\
\mathrm{X} \\
\end{array}$ & $\begin{array}{l}.906 \\
.849 \\
\end{array}$ & $\begin{array}{l}.897 \\
.771 \\
\end{array}$ & $\begin{array}{l}.494 \\
.724 \\
\end{array}$ & $\begin{array}{l}.931 \\
.846 \\
\end{array}$ \\
\hline $\begin{array}{r}\text { Milton } \\
\text { Pope }\end{array}$ & $\begin{array}{l}.799 \\
.756\end{array}$ & $\begin{array}{l}.714 \\
.653\end{array}$ & $\begin{array}{l}.646 \\
.789\end{array}$ & $\begin{array}{l}.869 \\
.772\end{array}$ & $\begin{array}{c}\mathrm{X} \\
.862\end{array}$ & $\begin{array}{c}.642 \\
\mathrm{X}\end{array}$ & $\begin{array}{l}.693 \\
.578\end{array}$ & $\begin{array}{l}.826 \\
.941\end{array}$ \\
\hline $\begin{array}{l}\text { RICHARDSON } \\
\text { SHAKESPEARE }\end{array}$ & $\begin{array}{l}.728 \\
.705\end{array}$ & $\begin{array}{l}.806 \\
.633\end{array}$ & $\begin{array}{l}.457 \\
.726\end{array}$ & $\begin{array}{l}.856 \\
.692\end{array}$ & $\begin{array}{l}.811 \\
.862\end{array}$ & $\begin{array}{l}.546 \\
.838\end{array}$ & $\begin{array}{c}\mathrm{X} \\
.494\end{array}$ & $\begin{array}{c}.711 \\
\mathrm{X}\end{array}$ \\
\hline
\end{tabular}

graduate English students learn that Shakespeare is followed by a host of 'Metaphysical' poets. The Augustan period in which Pope is situated is characterized by a concern with literary propriety: figures of speech must be used sparingly. Elegant and simple comparisons are favored. However, because the long poetic tradition is one of continuous reference and allusion, these findings are not, perhaps, so surprising. It is in the eighteenth century that Shakespeare is installed as England's national genius, after all. And in 1725 Pope published an edition of Shakespeare's works. ${ }^{7}$ Pope's annotators discover echoes of Shakespeare in almost all of his poems.

Likewise, scholarship on John Keats often draws connections between the Romantic poet's use of language and the poetry of Milton and Shakespeare. Many of these critical comparisons are motivated by formal investigations of Keats' poetry: Keats adapts early modern sonnet structures to write his own famous sequence of odes and borrows from Milton in his fragmentary attempts to write a modern epic. But our results demonstrate that Keat's figuration of mental substance and activity would also seem to exhibit continuity with those metaphors of mind used by Milton and Shakespeare. As in the case of Shakespeare's and Pope's use of metaphors, the classifier trained on Keats does well when predicting 
metaphors in Milton and Shakespeare. The critic interested in constructing genealogies and who happily explores influence or traces continuities in poetic diction will be amply rewarded by the results presented in Table 2. If a machine learns from one poet to find metaphors in another, we are encouraged to reopen discussions of what that latter poet may have learned from the former.

The discovery that a model transfers from one author to another is significant. The history of metaphors for the mind may include a long tradition of figures that are lightly altered to fit new contexts. Figurative resources employed in picturing the mind change slowly. Allusions add depth and the texture to a poem, and new poets quote older poets in order to insert themselves in the very tradition from which they draw their citations. A more cynical interpretation of these results, points out the underlying politics of canon formation: the transference between Pope and Shakespeare displays little more than the unified taste of those critics who determine just which (English, white, male, bourgeois) authors are to be installed in the pantheon of western literature. The foregoing are commonplaces of literary history, but they bear repeating in the current critical climate in which explorations of rupture are valued over descriptions of accretion and continuity.

\subsection{Testing Inter-Author Ambiguity}

While the preponderance of our examples are drawn from poetry, we also studied the metaphorics of two important eighteenth-century novelists, Henry Fielding and Samuel Richardson. It is well-known that Fielding's and Richardson's relationship was not a friendly one: Fielding's career as a writer of prose fiction began when he satirized Richardson's first novel. Richardson never forgave Fielding, even after Fielding complimented Richardson's Clarissa and composed his own Amelia in the serious, didactic style of Richardson. Fielding's novels are largely energetic, humorous, even sexy; Richardson's brim with sentiment and sentiments, and showcase nightmarish, claustrophobic interactions. And yet, the amount of transfer between models trained on these two authors shows that Fielding and Richardson do share metaphors of mind. 
Table 3: Confusion Matrix for Fielding vs. Richardson

\begin{tabular}{|c|c|c|c|c|}
\hline & \multicolumn{4}{|c|}{ PREDICTED } \\
\hline ACTUAL & $\begin{array}{c}\text { FiELDG. } \\
\text { Non-META. }\end{array}$ & $\begin{array}{c}\text { RichDSN. } \\
\text { Non-MetA. }\end{array}$ & $\begin{array}{c}\text { FIELDG. } \\
\text { META. }\end{array}$ & $\begin{array}{c}\text { RiCHDSN. } \\
\text { Meta }\end{array}$ \\
\hline FieldG. Non-Meta. & 34 & 14 & 14 & 1 \\
\hline Richdsn. Non-Meta. & 9 & 71 & 6 & 12 \\
\hline FieldG. MetA. & 5 & 13 & 79 & 36 \\
\hline Richdsn. Meta. & 7 & 24 & 38 & 90 \\
\hline
\end{tabular}

We take this analysis one step further, and produce a confusion matrix by group the data sets from the two authors and assigning four class labels to the metaphors: Fielding Metaphors, Fielding Non-Metaphors, Richardson Metaphors, and Richardson NonMetaphors. This data set was split into training and test sets with cross validation, and the results of multi-class SVM classification are shown in the resulting confusion matrix in Table 3. The results along the diagonal show the number of correctly classified metaphors. Of particular interest, however, are the relatively high number of times that an actual Fielding Metaphor was classified as a Richardson Metaphor and that an actual Richardson Metaphor was classified as a Fielding Metaphor. These results show that, whatever else separates them, the metaphorical vocabularies of these two authors do, indeed, overlap enough that they are difficult to distinguish one from the other.

Overall, the results from SVM classification show that automated expansion of the database is a practical alternative to pure manual effort. Although these methods produce high levels of accuracy, they are not free from error. One use of these methods is to employ them as an initial filter, to speed manual labeling efforts. But we must also consider the question of what level of noise (if any) is acceptable in such a collection. The literary historian is not pleased to find errors inserted into his database, but may well learn that noise and error are of interest in themselves. When a machine mistakes a metaphor, the example asks to be attended to more closely. ${ }^{8}$ 


\section{Blank Slates: a Case Study}

We hope to illustrate the practical utility of this project by pulling out a specific constellation of metaphors from the Mind is a Metaphor collection and discussing it in the context of literary history. Ours is a metaphor's- or meme's-eye view of discourse, and we take as an example, the best-known metaphor from British empiricism, an emblem of empiricism itself. In this section, we present a case study of Locke's 'white Paper, void of all Characters.'

\subsection{Overturning Misconceptions}

The most magisterial critic of eighteenth-century metaphors of mind, M.H. Abrams, claims in his classic study The Mirror and the Lamp, that it was John Locke, "who more than any philosopher established the stereotype for the popular view of the mind in the eighteenth century' (Abrams, 1953). Abram's assignment of Locke's place in intellectual history is taken for granted by most every practicing critic of eighteenth-century literature. But as will be demonstrated, the true history of Locke's metaphor is both more complicated and more compelling. By navigating electronic texts we put in question those commonplace from the history of ideas that have exaggerated Locke's place in the history of the eighteenth century.

The first thing revealed by harvesting and evaluating metaphors is that the metaphor both predates Locke's Essay Concerning Human Understanding (Locke, 1975) and outruns Locke's application of it. The tabula rasa of Aristotle, Proclus, and Aquinas survives the print revolution, and scraped wax tablets are updated, mutatis mutandis, as blank sheets of paper. The metaphor appears in several Renaissance courtesy manuals. In seventeenthcentury England two poets and playwright anticipate Locke's metaphor. Thomas Hobbes too describes 'common people's minds' as 'clean paper,' which is 'fit to receive whatsoever by public authority shall be imprinted in them.' Locke's metaphor obscures these earlier uses and others that follow. So Joseph Addison writes for a 'Set of Men' called the 'Blanks of Society,' Thomas Jefferson deploys the metaphor to characterize the continual revolution in American architecture, and in a Jacobin novel by Mary Hays, a misogynistic character 
asserts, 'The mind of a young lady should be clear and unsullied, like a sheet of white paper.'

More than a tabulation of uses, an aggregation of metaphors points up issues in semantics and the 'epidemiology' or 'phenomenology' of metaphor. We have found that Locke's refinement of the blank slate metaphor does not displace innatist figures of speech, as is commonly argued by historians of philosophy ${ }^{9}$. In fact, innate stamps and marks in the mind persist in eighteenth-century discourse in spite of Lockean metaphorics. It becomes clear, as we collect examples from electronic text collections, that Locke's deployment of the metaphor is only one of several competing uses of the metaphor. The use of the metaphor by Locke and other empiricist philosophers to make a case against the existence of innate characters or principles written in the soul is only one of many available uses of the metaphor. In the literature published in the period that follows the appearance of Locke's Essay in 1690, authors put the blank slate metaphor to several different, often contradictory uses. The metaphor is not univocal; it is ambiguous, polysemous. To treat the mind as a blank slate, without specifying a rhetorical context, is to invite any number of competing paraphrases of the metaphor, a great number of which have little or nothing to do with the existence of innate marks or characters written, 'by the finger of God,' in the infant mind or soul. ${ }^{10}$

\subsection{Context and Metaphor}

Because the history of ideas and computer-aided analysis of literature should never be pursued to the exclusion of social and cultural historical evidence, we look to the material culture and contemporary print technology for further clarification. The Stamp Acts passed by Parliament supply content to the metaphors. The eponymous stamp, a red mark placed on a sheet of paper, indicated that the tax had been paid (see Figure 3). Because much paper was stamped before it was put through a printing press, Locke's 'white Paper' may be pictured as already marked with a red stamp.

Locke's use of the metaphor is thus vitiated by its own print culture context and proves reversible, and Locke's antagonists are quick to reappropriate it, putting it to a use antithet- 


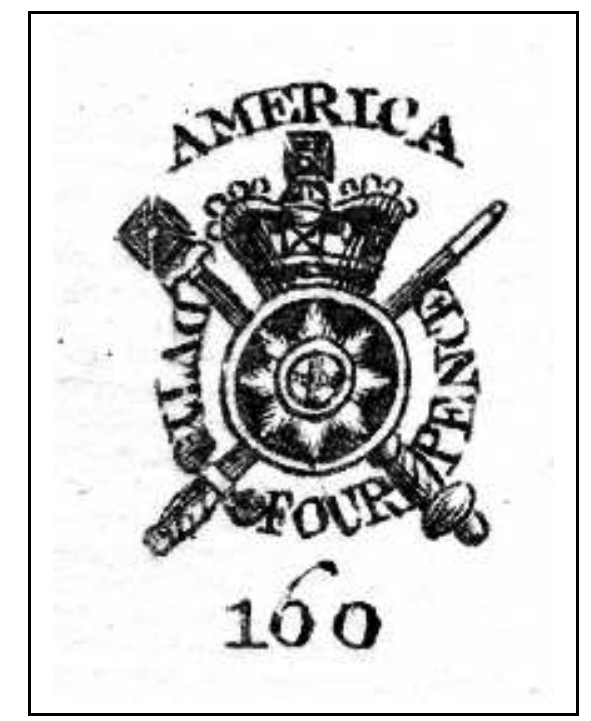

Figure 3: A Tax on the Blank Page.

ical to Locke's. The stamps incorporate an image of the monarch's crown, and the design of these stamps often includes the word 'DUTY' (as in a 'tax'), which suits them ideally to the ethical and theological projects of those philosophers and theologians who recruit metaphors of divine stamping in their revisions of Locke's 'white paper' metaphor and who theorize an innatist ethics of moral obligation or imagine an inborn faculty of moral sense.

While some twenty explicitly Lockean citations appear in the electronic text collections searched by keyword, more than half of these citations are negations, denials, or qualifications of the blank slate metaphor. The great majority of the almost two hundred instances of 'white paper' or 'tabula rasa' do not reference Locke's use of the metaphor at all. Often attribution is oblique: the mind has been compared to a tabula rasa by 'some,' 'some authors,' or 'some philosophers.' Epicurus is credited in many cases, Aristotle in many more. By mining the 'million book' library and harvesting metaphors from hundreds of books, we produce a more accurate and more nuanced picture of a metaphor's place in the history of philosophy. We discover that the blank slate is not Locke's metaphor; it only came to be associated with his philosophy so exclusively in later centuries. 
Throughout the eighteenth century the metaphor of the blank slate may be readily associated with Epicureans, Socinians, materialists, atheists, Free-Thinkers, and Hobbists, but the metaphor also continues to function in antagonistic devotional contexts where the sinner must prepare him or herself to receive the stamp or impress of divine truth by erasing, blotting, or removing all the false and world opinions that have been scribbled in his or her soul. In a number of sermons the sinner is exhorted to address himself to God with entire resignation: 'The Heart must be Tabula Rasa, white Paper to his Pen ... Let [God] write upon me what he pleaseth, and make what Impressions he pleaseth upon me' (20). Skeptics too use the metaphor in much the same way: the doubter's mind is a blank slate, without bias, expunged of opinion. In two particular cases, the metaphor is even associated with Descartes' philosophical project of hyperbolical doubt ${ }^{11}$ - the very philosophical project that many historians of philosophy credit Locke's argument against innate ideas with displacing.

And while Locke is often located in a tradition of radical Whig politics, the blank slate is not always an emblem of equality at birth and a call to reform and revolution. The metaphor is more ambivalent, if not fully ambiguous, and proves a ready-made emblem of oppression. Hobbes's common people's minds are available to be scribbled over with ideology, and male hegemony would preserve the fair, blank sex still clean and spotless until the blank young lady is ready for marriage. The blank slate metaphor is involved in pedagogical contexts but is also used to picture those geriatric minds in which all ideas have come to be erased. The metaphor is denied by some theologians but employed by others, it is found alternately in Pyrrhonist, neo-Aristotelian, and moral sense philosophy. Pedagogues of all stripes use the metaphor to forward specific, incompatible educational agendas. In short, the meaning of a metaphor is given by the context in which it appears. Traditional metaphors endure and prove reversible, antagonists put the same metaphor to antithetical purposes. The blank slate is, as it were, a blank slate. 
Table 4: Kullback-Liebler Analysis for Shakespeare Metaphors.

\begin{tabular}{|l|c|c|c|c|}
\hline \hline RANK & HEART & MIND & BODY & SOUL \\
\hline 1. & HEART & MIND & MY & SOUL \\
2. & MY & FORMD & QUENCH & MY \\
3. & STEEL & HAUE & UNLOAD & BLACK \\
4. & TEARTS & WEAKE & KINDLING & DON'T \\
5. & THY & OPPREST & BODY & GROSSLY \\
6. & BREAST & KINDES & MOISTURE & THOUGHTS \\
7. & QUENCH & IMPRESSION & FURNACE & LIM \\
8. & STUBBORN & MARBLE & COALS & STRUGGLING \\
9. & FLINT & STIFF & FLAMES & O \\
10. & TONGUE & GRIPED & SELFSAME & TRUNKS \\
11. & KINDLING & DROSS & FIRES & WING \\
12. & UNLOAD & FRAUD & BURNS & INFUSE \\
13. & HIS & STOOPS & SERVES & ANIMALS \\
14. & STRINGS & WAXEN & BURDEN & VESTURE \\
15. & FURNACE & EYE & SCARCE & HIS \\
\hline \hline
\end{tabular}

\section{Automating Metaphorical Analysis}

The preceding case study highlights the utility of creating a digital library of curated metaphors - it enables the literary historian to practice his or her craft with greater sophistication. However, manually mining this data still introduces potential for what the computer scientist recognizes as human biases into the analysis. In this section, we examine two automated methods of analyzing metaphors. The first ranks keywords by importance, using the Kullback-Liebler distance from information theory (Duda et al., 2000), which reveals underlying patterns of word usage, semantics, and style. The second method creates a hierarchical clustering of the metaphors, grouping similar metaphors together as branches of a tree, and makes pattern discovery possible at higher more abstract levels of interpretation.

\subsection{Kullback-Liebler Distance}

One question to ask is, what words are most strongly associated with metaphors of the mind? We conduct analysis to answer this question, using the Kullback-Liebler distance $\left(D_{K L}\right)$, which is a (non-metric) measure of distance between two probability distributions (Duda et al., 2000). Similar to the concept of mutual information, $D_{K L}$ is based off the 


\section{Table 5: Kullback-Liebler Analysis for Pope Metaphors.}

\begin{tabular}{|l|c|c|c|c|}
\hline \hline RANK & HEART & MIND & BODY & SOUL \\
\hline 1. & HEART & MIND & BODY & SOUL \\
2. & STEEL & RUL & SOUL & BODY \\
3. & BRAIN & SOUL & CAVE & MIND \\
4. & HER & BODY & CONFORMATIONS & OPERATIONS \\
5. & FEATHERS & PASSION & REFIN & STEELD \\
6. & CAELEST & FIND & ARTERIES & SELF \\
7. & TUN & PROPOSITIONS & NERVES & CAVE \\
8. & BODIED & ANARCHY & MIND & PINEAL \\
9. & NOSEGAY & FAINTLY & RECEPTACLE & GLAND \\
10. & MY & DANCING & ROUL & INVESTIGATE \\
11. & WINDOWD & GLIMMRING & OPERATIONS & ARTERIES \\
12. & IDEAS & CONFIND & PIND & FERMENTATION \\
13. & QUIVERS & JIGS & SEPULCHRE & FEVER \\
14. & SOUL & REFIN & SUPPOSD & RESTLESS \\
15. & KNOTS & VOMITION & SAVRY & QUIVERS \\
\hline \hline
\end{tabular}

notion of cross entropy between two distributions. The formula for $D_{K L}$ is:

$$
D_{K L}(P, Q)=\sum_{i} P(i) \log \frac{P(i)}{Q(i)}
$$

In our context, for each word $i$ in the lexicon of a given author, this measure compares the probability $P(i)$ of this word occurring in metaphorical usage by that author to the probability $Q(i)$ of that word occurring in general usage as measured across the full corpus of that author's text. Thus, words are weighted by how important they are to that author's metaphorical style.

We have measured the $D_{K L}$ for words appearing in Shakespeare's and Pope's metaphors of heart, mind, body, and soul. The fifteen most important words on this measure are reported in Tables 4 and 5 (stop words omitted). We found the encounter with a string of words ranked by Kullback-Liebler distance to be an alienating one for the literary historian, but quickly realized that just this sort of defamilarization is a good thing. At first a literary critic will fixate on those terms that he or can connect to a line in the poem from which it originates, but the critic eventually begins to learn to read these columns of words and numbers in lieu of the texts from which they were derived. The table then becomes a new 
text and presents itself for interpretation. Obviously, this is no ordinary form of literary interpretation, but the insights won from the tables and charts here displayed bear upon the more traditional evaluations and appraisals of a writer's use of language. The critic returns to the printed page with a new appreciation for the complex patterns operating almost invisibly in literary language. Specifically, the critic returns and pays closer attention to passages with the Kullback-Liebler words in them.

In the Kullback-Liebler tables historical and thematic differences between Pope and Shakespeare become visible again. While 'steel' ranks high in relation to 'heart' in both Shakespeare's and Pope's analyses, the Shakespearean heart includes 'flint' and consequent terms redolent of fire ('quench,' 'kindling, 'furnace'). Shakespeare would here seem to be in the thrall of both Galenic conceptions of the heart as the source of vital heat and traditional figures that picture love as a flame. Pope's terms are more motley: feathers, nosegays, and quivers appear. Shakespeare's heart is marked with the first person possessive; Pope's with the third person - not 'my' heart but 'hers.' Shakespeare's mind is opprest, stiff, and stoops; Pope's dances and glimmers but is prone to anarchy and vomition.

Pope would also seem to display advances in eighteenth-century brain science. Under the heading 'Body,' 'nerves' and 'arteries' rank high, under 'Soul,' 'pineal,' 'fever,' and 'gland.' Shakespeare's body is, like his heart, on fire: we picture kindling, coals, flames, fires, and burning. Pope, crippled by tuberculosis of the bone, lived as a 'hunchback' in a period that had no sympathy for any kind of physical deformity. The terms that rank high under 'Body' include 'Sepulchre.' Strong dualism is here apparent in Pope: the body is also a cave or a receptacle. At the head of the columns, 'mind,' 'body,' and 'soul' jostle for position.

\subsection{Hierarchical Clustering}

Examining word importance with the $D_{K L}$ score yields useful insights, but may not be enough to tell the whole story. For example, the top $D_{K L}$ words for the blank slate metaphors of the mind are 'tabula,' 'rasa,' 'blank,' and 'paper.' Clearly, all these words carry 


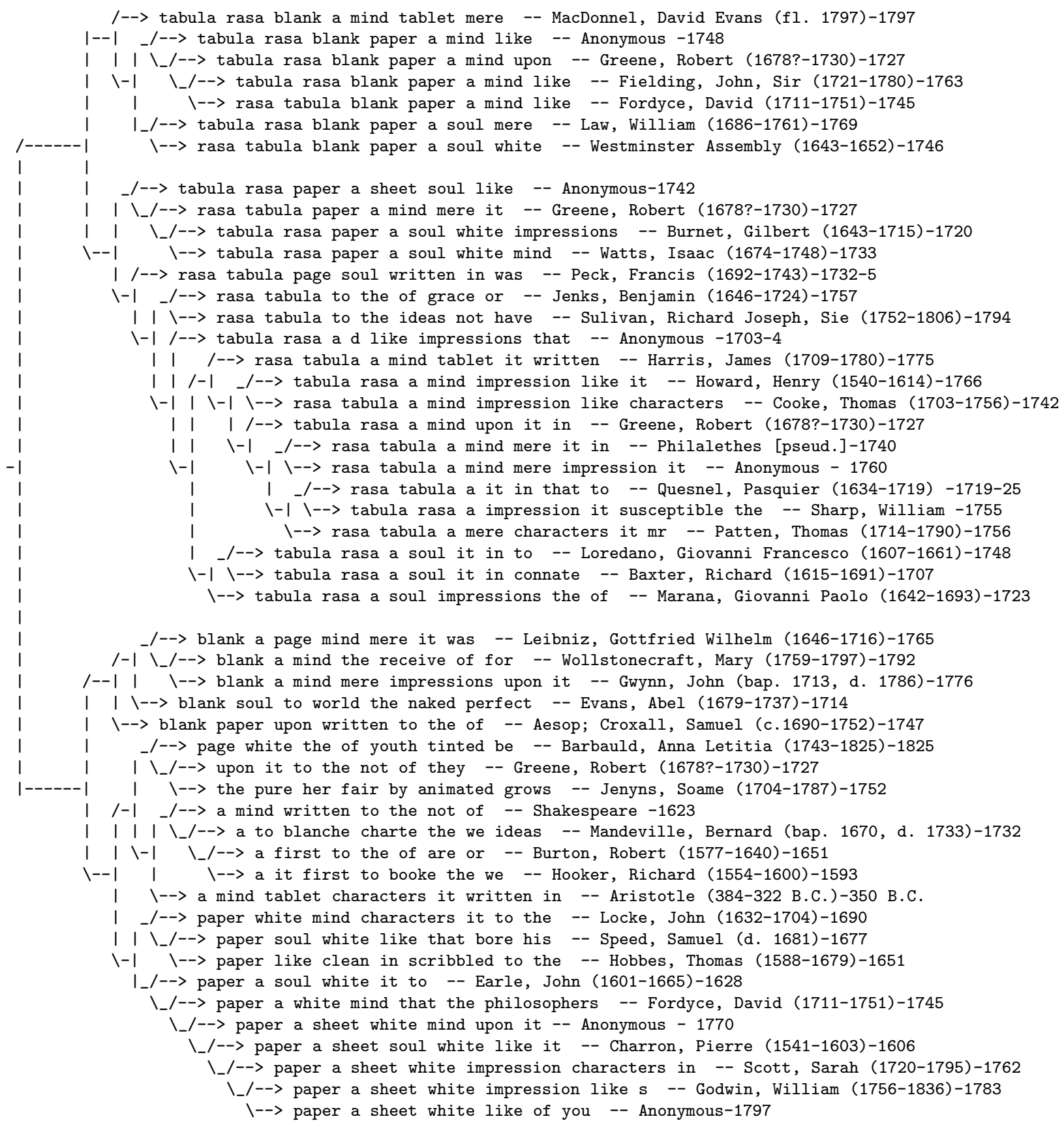

Figure 4: Hierarchical Clustering of Blank Slate Metaphors. This tree shows the results of hierarchical clustering on 50 metaphors randomly sub-sampled from the Blank Slate data set of 188 metaphors. (A complete hierarchical clustering on all 188 metaphors in the data set is available online at http://www.eecs.tufts.edu/ dsculley/metaphors/blankSlate.all.cluster.txt ) This automated clustering reveals several of the trends regarding blank slate metaphors of the mind detailed in the case study. 
importance, but from a list alone it is unclear how they interacted in eighteenth-century blank slate metaphors of the mind. A deeper level of automated analysis is required, and for this we turn to clustering methods.

There are a wide variety of clustering techniques; these belong to the family of unsupervised machine learning methods. In general, clustering seeks to automatically group similar examples, where similarity is defined by some distance measure. (See the text by Duda et al. (2000) for a complete discussion of clustering methods.) In this analysis, we apply a hierarchical clustering method to all of the collected blank slate metaphors, which allows clear visualization of the patterns of usage.

The particular clustering method we applied creates a hierarchical tree of examples by iteratively joining the two most similar examples in the data set (Stolcke, 1996). (This is referred to as the "unweighted pair group" method by Sneath and Sokal (1973)). Distance between metaphors was computed by mapping each metaphor to a bag of words vector, in which the score for each feature was assigned by $x_{i}=b(i) D_{K L}(m(i), n(i))$, where $b(i)$ is the binary $\{0,1\}$ score indicating presence of word $i$ in the given metaphor, $m(i)$ is the probability of that word occuring in metaphorical usage, and $n(i)$ is the probability of the word occuring in the author's general usage. We use $D_{K L}$ term weighting rather than other scoring schemes, such as binary weighting or TF-IDF scoring, as we this method computes similarity between metaphors based on the strength of the metaphorical keywords in the examples.

The results of hierarchical clustering on blank slate metaphors are given in Figure 4. The hierarchical clustering reveals two main clusters of the blank slate metaphors, those using the tabula rasa metaphor of the mind, and those using the blank sheet metaphor. Additionally, there are several small clusters in which both the tabula rasa and the blank slate metaphors are used together. Each cluster might be understood as a specific metaphor, the whole as a constellation exhibiting close family resemblance. Clustering metaphors, we see how terms become synonymous. The scraped wax tablets of antiquity are updated and survive in poetic diction. The metaphor survives changing print technologies; slates are replaced 
by sheets of paper. In spite of the changing the material, print-culture environment, the metaphor persists.

Hierarchical clustering is one method of automating pattern visualization and discovery in the library of metaphors. Extending this work in the direction of author-topic modeling (Rosen-Zvi et al., 2004) as applied to metaphors may allow pattern discovery and visualization across much larger subsets of this collection.

\section{Future Work: Increasing Automation and Collaboration}

As has been intimated throughout this paper, it is our intention to collect metaphors from a variety of historical periods. A new, open, interdisciplinary site will grow from the core set of metaphors of mind that was collected over the past three years. These metaphors constitute the foundation of the project, but over time the metaphor collection will continue to grow. Most profitably, the first additions to the site would include metaphors of mind harvested from the periods just preceding and following the eighteenth century. As the collection of metaphors expands, the website would become a destination for researchers from every imaginable discipline. A more complete collection of metaphors would attract an even more diverse stream of visitors.

\subsection{Automated Harvesting}

The current database of metaphors has been assembled through manual effort over a number of years. Machine learning methods can capitalize on this high quality training data to automate the process of harvesting a much larger set of metaphors. The current database should be sufficient to exhaust the eighteenth-century metaphors of the mind available in Chadwyck-Healey's electronic collection of English literature.

We believe that expanding the database may be accomplished by beginning with the neighboring historical periods, such as the seventeenth and nineteenth centuries and relying on our models to transfer. In this situation, we expect that the methods of semi-supervised learning may provide strong benefit. In semi-supervised learning, a small amount of labeled 
training data is used in conjunction with a large amount of unlabeled training data - in effect, combining the benefits of supervised classification and unsupervised clustering (Zhu, 2007). These methods will provide leverage for our relatively small seed data set.

However, if we attempt to expand the database of metaphors into new eras, such as cyberpunk metaphors of the mind, or across many languages, it is unlikely that our current database will yield useful training data. In this case, additional manual labeling effort will be required. The learning curves shown in Section 3 showed that this effort may not be prohibitive - perhaps as few as one or two hundred examples may be enough to begin work in a new field. We may be able to reduce this initial effort even further through the use of active learning, in which a machine learning classifier identifies examples for which manual labeling will give the most benefit (Cohn et al., 1995). Previous applications of active learning have been effective in reducing the amount of manual labeling necessary to reach asymptotic classification accuracy, in a number of fields including text classification (Tong and Koller, 2002). We will find out if it can give similar benefit in our metaphorical setting.

\subsection{Unsupervised Analysis}

When and how does a vital, poetic metaphor harden into a few established uses? When, for example, does Locke's name come to be finally and ineluctably associated with the tabula rasa? What would an exhaustive history of the blank slate - or the mind's eye or metaphors of interiority - in English literature even look like? Will Chaucer's metaphors transfer to Joyce's novels? These are questions that cannot be answered by one unassisted scholar. In the long run, manual hunt-and-peck methods will not do.

Unsupervised methods of pattern recognition may be applied to this task, and may benefit the literary analyst. Hierarchical clustering is one method, but we may also apply clustering methods such as Expectation Maximization (Duda et al., 2000) as in authortopic modeling (Rosen-Zvi et al., 2004) for additional benefit across wider scales. Methods of anomaly detection may help the researcher cull unusual or surprising metaphors hidden in 
the haystack. Finally, additional methods of visualization, such as temporal or geographic mapping, may enable help to show how metaphors and ideas moved through time and space. These are the tools that transform a database of metaphors into a digital library of metaphors. Moving forward, these tools may be of particular benefit for analyzing and comparing authors not currently in the canon, on a large scale across thousands or millions of texts. This automated analysis can be performed based on direct textual comparisons, rather than being restricted to superficial labelings such as religion, gender, or political affiliation.

It is important to avoid the illusion that automated analysis is somehow more objective or less biased than traditional methods. There is no new infallible science of literature forthcoming. As the "No Free Lunch" Theorem states (Wolpert and Macready, 1995), every machine learning method requires the acceptance of base level assumptions, such as the appropriate choice of distance metric or the shape of the probability distribution underlying the data. These assumptions must, at some level, be taken on faith, and influence the results of automated analysis, just as cultural and theoretical biases influence traditional analysis.

\subsection{Metaphorical Community}

We are interested in the opportunities for further interdisciplinary collaboration enabled through online community involvement and automation with human assistance. Our choice of metaphors of mind is a studied one, as some concept of person (if not conceptions of mind or soul) should be readily located in most cultures and historical periods. Where histories of the self have almost intrinsic interest for the self-reflective humanities, the digital humanities, as here practiced, promise wide-ranging investigations of linguistic transfer, convergence, divergence, and rupture. Cognitive and computational linguists, rhetoricians, literary critics, intellectual historians, psychologists, philosophers, and neuroscientists would all profit from browsing through a long, deep history of metaphors for the mind. A library is not just 
a collection of books - nor even a collection of metaphors - but is also a meeting place for researchers to come together and share ideas, questions, thoughts, and conversations.

\section{Notes}

${ }^{1}$ Chadwyck-Healey, the main text collection drawn upon, is accessed through a custom search interface
hosted at Stanford University Library. The search interface is called HUGO. It is a system for browsing and searching texts encoded in Standard Generalized Markup Language (SGML). Stanford digital librarian Glen Worthey informs us that the principal programmer, Rick Wong, named the program after Hugo de St. Caro, or Cardinal Hugo, compiler of the first concordance. A brief history of HDIS is available at http://library.stanford.edu/depts/hasrg/hdis/about.html. HDIS's main British and Irish literature resource page is available at http://www-sul.stanford.edu/depts/hasrg/hdis/text-brit.html. ECCO may be found at http://galenet.galegroup.com/servlet/ECCO; EEBO at http://eebo.chadwyck.com/home.

${ }^{2}$ http://www.cs.bham.ac.uk/ jab/ATT-Meta/Databank

${ }^{3}$ http://www1.uni-hamburg.de/metaphern/index_en.html

${ }^{4} \mathrm{http}: / / \operatorname{cogsci}$. berkeley.edu/lakoff

${ }^{5}$ Barnden has collected 1,070 'manifestations' of metaphor from texts and 65 additional speech examples

${ }^{6}$ Although we do not find the irregular spellings of this period to have significant impact in our experiments, the problem of canonicalizing irregular spellings is an area for interesting future work. Martin Mueller is currently exploring hand-tuned approaches for this task; algorithmic 'spell checking' approaches using either string-based or phoneme-based edit distances are also possible.

${ }^{7}$ On the Shakespearean aspects of Pope's poetry, see, for example, Debra Leissner, 'Pope, Petrarch, and Shakespeare: Renaissance Influences in "Eloisa to Abelard."' Philological Quarterly vol. 74. 1995. Because turnabout is fair play and the models transfer in both directions, we might just as well cite Catherine Bates' 'Pope's Influence on Shakespeare?' Shakespeare Quarterly. 1991. pp. 57-9.

${ }^{8}$ We take seriously Stephen Ramsay's repeated recommendation to pay attention to the anomalies. See, for example, his insistence that curious anomalies may 'appear to conform to some of the more famous critical statements' in certain interesting cases. Stephen Ramsay, 'In Praise of Pattern.' TEXT Technology. Number 2, 2005. p. 188.

${ }^{9}$ See, for example, the following canonical statement by Kenneth Maclean: 'The literature immediately following Locke ... abounds with notions of the mind as a tabula rasa, with democratic conceptions of mental equality, and with the idea of ruling passions, each of which owes it rise to Locke's denial of innate ideas;' in John Locke and English Literature of the Eighteenth Century. New York: Russell \& Russell, Inc., 1962. pp. $20-1$. 


\footnotetext{
${ }^{10}$ Locke's argument against innate ideas is addressed to a host of seventeenth-century Rationalists, Cambridge Platonists, and theologians. A compressed account of Locke's metaphor and its place in the context of the seventeenth-century philosophy is available in John W. Yolton, A Locke Dictionary, Blackwell Philosopher Dictionaries (Oxford, UK ; Cambridge, Mass., USA: Blackwell, 1993) 288-9.

${ }^{11}$ In the preface of a translation of Giovanni Marana's popular Turkish Spy the blank slate is associated with the philosophy of René Descartes. Where Gassendi and Locke employ the blank slate metaphor in order to qualify or overturn a Cartesian model of the mind, Marana details how the Cartesian practice of hyperbolical doubt cleans and polishes the soul so that it becomes a 'Tabula Rasa' (Preface). Moreover, Marana is not alone in his association of Cartesian philosophy with the tabula rasa. In the entry on John Maldonat in an eighteenth-century English translation of Pierre Bayle's Dictionary, we find the following assertion: 'We must examine every thing, as if we were a tabula rasa. This is doubtless what Des Cartes intended, when he would have his Philosopher doubt of every thing, before he examines the reasons of the certainty of it" (vol. iv, 81).
}

\section{References}

M. H. Abrams. The Mirror and the Lamp: Romantic Theory and the Critical Tradition. Oxford University Press, 1953.

Aristotle. The Poetics. Introduction to Aristotle. 2nd ed. R. McKeon, Ed. and Trans. U of C Press, 1973.

J. L. Austin. A plea for excuses. Philosophical Papers, pages 175-204, 1961.

C. Bates. Pope's influence on shakespeare? Shakespeare Quarterly, 1991.

C. J. C. Burges. A tutorial on support vector machines for pattern recognition. Data Mining and Knowledge Discovery, 2(2):121-167, June 1998.

C. Chang and C. Lin. LIBSVM: a library for support vector machines, 2001. Software available at http://www.csie.ntu.edu.tw/ cjlin/libsvm.

D. Cohn, Z. Ghahramani, and M. Jordan. Active learning with statistical models. In G. Tesauro, D. Touretzky, and T. Leen, editors, Advances in Neural Information Processing Systems, volume 7, pages 705-712. The MIT Press, 1995. 
G. Crane. What do you do with a million books? D-Lib Magazine, 17(3), 2006.

N. Cristianini and J. Shawe-Taylor. An introduction to support vector machines. Cambridge University Press, 2000.

E. R. Curtius. European Literature and the Latin Middle Ages, Bollingen Series XXXVI. Princeton University Press, 1990.

R. Duda, P. Hart, and D. Stork. Pattern Classification (2nd Edition). Wiley-Interscience, 2000.

Thorsten Joachims. Text categorization with suport vector machines: Learning with many relevant features. In ECML '98: Proceedings of the 10th European Conference on Machine Learning, pages 137-142, 1998. ISBN 3-540-64417-2.

S. Johnson. A Dictionary of the English Language: In Which the Words Are Deduced from Their Originals, and Illustrated in Their Different Significations by Examples from the Best Writers. To Which Are Prefixed, a History of the Language, and an English Grammar. AMS Press, 1967.

G. Lakoff and M. Johnson. Metaphors we Live By. University of Chicago Press, 1980.

D. Leissner. Pope, petrarch, and shakespeare: Renaissance influences in 'eloisa to abelard. Philological Quarterly, 74, 1995.

J. Locke. An Essay Concerning Human Understanding. Oxford University Press, 1975.

R. Lonsdale, editor. Eighteenth-Century Women Poets. Oxford University Press, 1989.

K. Maclean. John Locke and English Literature of the Eighteenth Century. Russell \& Russell, Inc, 1962.

T. M. Mitchell. Machine Learning. McGraw-Hill, 1997.

B. Pasanek. Eighteenth century metaphors of the mind, a dictionary, 2006. 
S. Ramsay. In praise of pattern. TEXT Technology, (2), 2005.

M. Rosen-Zvi, T. Griffiths, M. Steyvers, and P. Smyth. The author-topic model for authors and documents. In AUAI '04: Proceedings of the 20th conference on Uncertainty in artificial intelligence, pages 487-494, 2004.

G. Salton and C. Buckley. Term-weighting approaches in automatic text retrieval. Inf. Process. Manage., 24(5):513-523, 1988.

B. Scholkopf and A. Smola. Learning with Kernels. MIT Press, 2002.

P. Sneath and R. Sokal. Numerical taxonomy; the principles and practice of numerical classification. W. H. Freeman, San Francisco, 1973.

A. Stolcke. Cluster 2.9, 1996. Available at http://www.icsi.berkeley.edu/ftp/global/ pub/ai/stolcke/software/cluster-2.9.tar.Z.

S. Tong and D. Koller. Support vector machine active learning with applications to text classification. Journal of Machine Learning Research, 2:45-66, 2002.

I. H. Witten and E. Frank. Data Mining: Practical Machine Learning Tools and Techniques, 2nd ed. Morgan Kaufman, 2005.

D. H. Wolpert and W.G. Macready. No free lunch theorems for search. Technical report, Santa Fe Institute, Santa Fe, NM, USA, 1995.

J. W. Yolton. A Locke Dictionary. Blackwell Philosopher Dictionaries, 1993.

X. Zhu. Semi-supervised learning literataure survey. Technical Report 1530, University of Wisconsin-Madison, 2007. 